International Journal of Pure and Applied Mathematics

Volume 112 No. 2 2017, 277-283

ISSN: 1311-8080 (printed version); ISSN: 1314-3395 (on-line version)

url: http://www.ijpam.eu

doi: $10.12732 /$ ijpam.v112i2.5

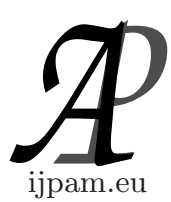

\title{
A NOTE ON SPECTRUM PRESERVING ADDITIVE MAPS BETWEEN $C^{*}$-ALGEBRAS
}

\author{
Rohollah Parvinianzadeh \\ Department of Mathematics \\ University of Yasouj \\ P.O. Box 75918-74831, Yasouj, IRAN
}

\begin{abstract}
Let $A$ be a unital $C^{*}$-algebra of real rank zero and $B$ a unital semi-simple Banach algebra. Let $\phi$ be a surjective spectrum preserving additive map from $A$ onto $B$. Then $\phi$ is continuous and Jordan isomorphism on self-adjoint element of $A$.
\end{abstract}

AMS Subject Classification: 46H05, 47B48, 47A10

Key Words: Banach algebra, $C^{*}$-algebra of real rank zero, spectrum preservingg map, von Neumann algebra

\section{Introduction}

All algebras we deal with in this paper are complex and unital. The identity element of an algebra $A$ will be denoted by $e$, or $e_{A}$, for distinction. For a given algebra $A$ and $a \in A, \sigma(a)$ will denote the spectrum of $a$. Given two algebras $A, B$, a map $\phi: A \rightarrow B$ is called spectrum preserving if $\sigma(\phi(a))=\sigma(a)$ for every $a \in A$. Furthermore, $\phi$ is said to be unital if $\phi\left(e_{A}\right)=e_{B}$ and it is called invertibility preserving if $\phi(a)$ is invertible in $B$ whenever $a$ is invertible in $A$. Spectrum preserving linear mappings were studied for the first time by G. Frobenius [7]. He proved that a surjective linear mapping $\phi: M_{n}(\mathbb{C}) \longrightarrow$ $M_{n}(\mathbb{C})$ which preserves the spectrum has one of the forms $\phi(T)=A T A^{-1}$ or $\phi(T)=A^{t} T A^{-1}$ for some invertible $A$.

Received: September 11, 2016

Revised: $\quad$ December 14, 2016

Published: $\quad$ February 1, 2017 (c) 2017 Academic Publications, Ltd. url: www.acadpubl.eu 
Kaplansky [11] asked the below problem:

Let $A$ and $B$ be two semi-simple complex Banach algebras and let $\phi$ be a spectrum preserving linear mapping from $A$ onto $B$. Is it true that $\phi$ is Jordan isomorphism between $A$ and $B$ ?

This problem was motivated by the well-known Gleason-Kahane-Zelazko theorem [12], [10], [17], which constitutes a positive answer to the preceding problem in the commutative case. (The Gleason-Kahane-Zelazko Theorem says: Every unital invertibility preserving linear functional on a unital complex Banach algebra is necessarily multiplicative). Positive answers in the non-commutative case are due to B. Aupetit [1] for von Neumann algebras and to A.R. Sourour [16] for the algebra $B(X)$ of bounded linear operators on a Banach space $X$. This problem is still unsolved. The most important partial results obtained in this direction are [3], [4], [9], [16].

In [9] Jafarian and sourour proved that a surjective linear map preserving spectrum from $B(X)$ onto $B(Y)$ is either an isomorphism or an antiisomorphism where $X$ and $Y$ are complex Banach spaces and $B(X)$ is the Banach algebra of all bounded linear operators acting on $X$. In [14] Omladic and Semrl proved that the conclusions Jafarian and sourour [9] is valid under the less restrictive condition that map $\phi$ is only additive.

Recently Aupetit [1] showed that a spectrum preserving surjective linear map from a Von Neumann algebra onto another is a Jordan isomorphism. In [8] Jafarian asked any spectrum-preserving additive map from a Von Neumann algebra onto another is a Jordan isomorphism.

In this paper we prove that if $A$ be a unital $C^{*}$-algebra of real rank zero and $B$ a unital semi-simple Banach algebra. Let $\phi$ be a surjective spectrum preserving additive map from $A$ onto $B$. Then $\phi$ is continuous and Jordan isomorphism on self-adjoint element of $A$. Also, we obtain some interesting results in direction.

The following results will be often used in the sequel.

Lemma 1.1. [6, Lemma 5.1.5] Let $(A,\|\|$.$) be a unital Banach algebra,$ let $a \in A$, and let $\epsilon>0$. Then there is a norm $\||\cdot \||$ on $A$ such that $\| .\|\|$ is equivalent to $\|\cdot\|$, and $\||e \||=1$, and $\||a \|| \leq r_{A}(a)+\epsilon$.

Lemma 1.2. $[15]$ Let $A$ be a Banach algebra, let $p \in A[\mathbb{C}]$, where $A[\mathbb{C}]$ denote the algebra of all polynomials with coefficients in $A$. Then

$$
r_{A}(p(1))^{2} \leq \sup _{|z|=R} r_{A}(p(z)) \cdot \sup _{|z|=\frac{1}{\mathrm{R}}} r_{A}(p(z))
$$

for every $R>1$. 
Theorem 1.3. [1, Theorem 1.1] An element $p$ of a semi-simple complex Banach algebra $A$ is idempotent if and only if $\sigma_{A}(p) \subseteq\{0,1\}$ and there exist real numbers $r>0$ and $C>0$ such that

$$
\sigma_{A}(a) \subseteq \sigma_{A}(p)+c\|a-p\|
$$

for all $a \in A$ with $\|a-p\| \leq r$.

Definition 1.4. Let $A$ and $B$ be two unital Banach algebras. Let $\phi: A \rightarrow$ $B$ be a additive map. Then the separating space of $\phi$ is $C(\phi)=\{b \in B$ : there exists $a_{n} \rightarrow 0$ in $A$ with $\phi\left(a_{n}\right) \rightarrow b$ \}.

It is clear that $\phi$ is continuous if and only if $C(\phi)=\{0\}$.

\section{Main Results}

Let $A$ be a $*$-algebra, we say elements $a, b$ of $A$ are unitary equivalent if there exists a unitary element $u \in A$ such that $b=u a u^{*}$. Note that $\sigma_{A}(a)=\sigma_{A}(b)$ if $a$ and $b$ are unitary equivalent, [13, p. 36].

Theorem 2.1. Let $A$ be a Banach $*$-algebra and $B$ be semi-simple Banach algebra and let $\phi: A \rightarrow B$ be a surjective spectrum preserving additive map and $b \in C(\phi)$, where $C(\phi)$ is separating space of $\phi$. Then we have:

(i) $\phi$ is injective and $\phi\left(e_{A}\right)=e_{B}$.

(ii) $r_{B}(\phi(a)) \leq r_{B}(\phi(a)-b)$, for every $a \in A$.

(iii) $r_{B}(b)=0$ and $\phi$ is continuous.

Proof. (i) see [1, Proposition 2.1 (i) and (ii)].

(ii) Let $\left(a_{n}\right)$ be in $A$ with $a_{n} \rightarrow 0$ and $\phi\left(a_{n}\right) \rightarrow b$ as $n \rightarrow \infty$, and take $a \in A$.

For each $\epsilon>0$, we may, by Lemma 1.1, choose norms on $A$ and $B$ which are equivalent to the given norms and which are such that

$$
\|a\| \leq r_{A}(a)+\epsilon,\|\phi(a)-b\| \leq r_{B}(\phi(a)-b)+\epsilon \quad(a \in A, b \in B) .
$$

We apply Lemma 1.2 in the case where $p=\left(\phi(a)-\phi\left(a_{n}\right)\right)+\left(\phi\left(a_{n}\right)\right) \mathbb{C}$ be long to $B[\mathbb{C}]$, where $n \in N$. We have $p(1)=\phi(a)$, and so, for each $R>1$,

$$
r_{B}(\phi(a))^{2} \leq \sup _{|z|=R} r_{B}(p(z)) \cdot \sup _{|z|=\frac{1}{\mathrm{R}}} r_{B}(p(z)) \quad(*) .
$$


Now $r_{B}(p(z)) \leq\left\|\phi(a)-\phi\left(a_{n}\right)\right\|+|z|\left\|\phi\left(a_{n}\right)\right\|$. Since $\phi$ is onto, for every $n \in N$, there exists $x_{n} \in A$ such that $\phi\left(x_{n}\right)=z \phi\left(a_{n}\right)$. We have $p(z)=\phi(a)-\phi\left(a_{n}\right)+$ $z \phi\left(a_{n}\right)=\phi\left(a-a_{n}+x_{n}\right)$, and so, by hypothesis,

$$
r_{B}(p(z))=r_{B}\left(\phi\left(a-a_{n}+x_{n}\right)\right)=r_{A}\left(a-a_{n}+x_{n}\right) \leq\left\|a-a_{n}\right\|+\left\|x_{n}\right\| .
$$

Thus, from $(*)$, we have

$$
r_{B}(\phi(a))^{2} \leq\left(\left\|a-a_{n}\right\|+\left\|x_{n}\right\|\right)\left(\| \phi(a)-\phi\left(a_{n} \|+\frac{\| \phi\left(a_{n} \|\right.}{R}\right) .\right.
$$

This holds for each $n \in N$, and so, letting $n \rightarrow \infty$, we see that

$$
r_{B}(\phi(a))^{2} \leq\left(\|a\|+\left\|x_{n}\right\|\right)\left(\|\phi(a)-b\|+\frac{b}{R}\right) .
$$

Note that $x_{n} \rightarrow 0$, since

$$
\sigma_{A}\left(x_{n}\right)=\sigma_{B}\left(\phi\left(x_{n}\right)\right)=\sigma_{B}\left(z \phi\left(a_{n}\right)\right)=z \sigma_{A}\left(a_{n}\right)=\sigma_{A}\left(z a_{n}\right) .
$$

Hence $x_{n}$ and $z a_{n}$ are unitary equivalent, so that for every $n \in N$, there exists an unitary element $u_{n} \in A$ such that $x_{n}=u_{n} a_{n} u_{n}^{*}$. It follows that $\left\|x_{n}\right\| \leq$ $\left\|u_{n}\right\|\left\|a_{n}\right\|\left\|u_{n}^{*}\right\| \rightarrow 0$, since $a_{n} \rightarrow 0$. Therefore $x_{n} \rightarrow 0$. So

$$
r_{B}(\phi(a))^{2} \leq\|a\|\left(\|\phi(a)-b\|+\frac{b}{R}\right) .
$$

But This holds for each $R>1$, and so, letting $R \rightarrow \infty$, we obtain,

$$
r_{B}(\phi(a))^{2} \leq\|a\|\|\phi(a)-b\| \leq\left(r_{A}(a)+\epsilon\right)(r(\phi(a)-b)+\epsilon) .
$$

Finally this holds for each $\epsilon>0$, and so $r_{B}(\phi(a))^{2} \leq r_{A}(a) r_{B}(\phi(a)-b)$, for every $a \in A$.

Since $r_{A}(a)=r_{B}(\phi(a))$, for every $a \in A$, we have

$$
r_{A}(a) r_{B}(\phi(a))=r_{B}(\phi(a))^{2} \leq r_{A}(a) r_{B}(\phi(a)-b),
$$

for every $a \in A$, and so $r_{B}(\phi(a)) \leq r_{B}(\phi(a)-b)$.

(iii) Since $\phi$ is onto, there exists $t \in A$ such that $\phi(t)=b$. In (ii) taking $a=t$ we get $r_{B}(b)=0$.

Now we prove that $\phi$ is continuous, taking $x=t+a$ for every $a \in A$. By (ii) we have $r_{B}(b+\phi(a)) \leq r_{B}(\phi(a))$ for every $a \in A$. Hence $r_{B}(b+q)=0$ for every quasi-nilpotent element $q \in B$. By Zemanek's characterization of the radical [2, Theorem 5.3.1], $b \in \operatorname{rad}(B)=\{0\}$, and so $b=0$. Therefore $\phi$ is continuous.

A additive map is said to be idempotent preserving if it maps every idempotent to an idempotent. 
Theorem 2.2. Let $A$ be a Banach *-algebra and $B$ a semi-simple Banach algebra and let $\phi: A \rightarrow B$ be a surjective spectrum preserving additive map. Then $\phi$ is $\mathbb{R}$-linear and idempotent preserving.

Proof. Evidently every additive map between two algebra is $\mathbb{Q}$-linear. Let $a \in A$ and $r \in \mathbb{R}$. Choose sequence $\left\{q_{n}\right\}_{n=1}^{\infty} \subseteq \mathbb{Q}$ with $\lim q_{n}=r$ as $n \rightarrow$ $\infty$. Then $\lim q_{n} a=r a$, we obtain that $\phi(r a)=\phi\left(\lim q_{n} a\right)=\lim \phi\left(q_{n} a\right)=$ $\lim q_{n} \phi(a)=r \phi(a)$. Hence $\phi$ is $\mathbb{R}$-linear. Now we prove that $\phi$ is idempotent preserving. Let $p=p^{2}$ be an idempotent element of $A$. If $p=0$ then $\phi(p)=0$, and also $p=e_{A}$ then $\phi\left(e_{A}\right)=e_{B}$ are idempotents in $B$. So suppose $p \notin\left\{0, e_{A}\right\}$, then $\sigma_{A}(p) \subseteq\{0,1\}$. By Theorem 1.3, there exist real numbers $r>0$ and $c>0$ such that

$$
\sigma_{A}(a) \subseteq \sigma_{A}(p)+c\|a-p\| \quad\|a-p\|<r .
$$

Since $\phi$ is spectrum preserving by Theorem 2.1, maps $\phi, \phi^{-1}$ are continuous, consequently,

$$
\sigma_{B}(\phi(a)) \subseteq\{0,1\}+\frac{c}{\alpha}\|\phi(a)-\phi(p)\|,
$$

since the mapping $\phi$ is onto, we obtain

$$
\sigma_{B}(b) \subseteq\{0,1\}+\frac{c}{\alpha}\|b-\phi(p)\|
$$

for $b \in B$ and $b$ near to $\phi(p)$. Hence, Theorem 1.3, implies that $\phi(p)$ is an idempotent element of $B$.

It is easily seen that an idempotent preserving additive map must send a set of mutually orthogonal idempotents to a set of mutually orthogonal idempotents (two idempotents $p_{1}$ and $p_{2}$ are orthogonal if $p_{1} p_{1}=p_{2} p_{1}=0$ ).

We recall that a $C^{*}$-algebra $A$ is of real rank zero if the set of all real linear combinations of orthogonal self-adjoint idempotents is dense in the set of all self-adjoint elements of A, [5]. It is clear that every von Neumann algebra is a $C^{*}$-algebra of real rank zero. In particular, $B(H)$, the algebra of all bounded linear operators on a complex Hilbert space, has real rank zero.

Theorem 2.3. Let $A$ be a unital $C^{*}$-algebra of real rank zero and $B$ a unital semi-simple Banach algebra. Let $\phi: A \rightarrow B$ be a surjective spectrum preserving additive map. Then $\phi$ is a Jordan isomorphism on self-adjoint element.

Proof. Let $a \in A$ such that $a^{*}=a$ which is a real linear combination of orthogonal self-adjoint idempotents, i.e., $a=\sum_{i=1}^{n} r_{i} p_{i}$ with $r_{i} \in \mathbb{R}$ and $p_{i}^{2}=$ $p_{i}=p_{i}^{*}$ and $p_{i} p_{j}=p_{j} p_{i}$ if $i \neq j$. $\phi$ is continuous and maps mutually orthogonal 
Hermitian idempotents to mutually orthogonal idempotents by Theorem 2.1 and Theorem 2.2. So $\phi\left(a^{2}\right)=\phi(a)^{2}$. Now, since $A$ is a $C^{*}$-algebra of real rank zero, the set of self-adjoint elements, which are finite real linear combinations of orthogonal self-adjoint idempotents, is dense in the set of all self-adjoint elements in $A$, we see that $\phi\left(a^{2}\right)=\phi(a)^{2}$ hold for every self-adjoint elements $a$.

Remark 2.4. Since every von Neumann algebra is a $C^{*}$-algebra of real rank zero, so Theorem 2.3, is valid if $A$ be a von Neumann algebra

\section{References}

[1] B. Aupetit, Spectrum-preserving linear mappings between Banach algebras or Jordan-Banach algebras, J. London. Math. Soc., 62 (2000), 917-924, doi: $10.1112 / \mathrm{S} 0024610700001514$.

[2] B. Aupetit, A Primer on Spectral Theory, Springer, New York, 1991.

[3] B. Aupetit and H. du Toit Mouton, Spectrum preserving linear mappings in Banach algebras, Studia Math., 109 (1994), 91-100.

[4] M. Bresar, P. Semrl, Linear maps preserving the spectral radius, J. Funct. Anal., 142 (1996), 360-368, doi: 10.1006/jfan.1996.0153.

[5] L.B. Brown, G.K. Pedersen, $C^{*}$-algebras of real rank zero, J. Funct. Anal., 99 (1991), 131 149, doi: 10.1016/0022-1236(91)90056-B.

[6] H. G. Dales, P. Aiena, J. Eschmeier, K. Laursen, G. Willis, Introduction to Banach Algebras, Operators, and Harmonic Analysis, London Mathematical Society Monographs, 57, Cambridge university Press 2003.

[7] G. Frobenius, Über die Darstellung der endlichen Gruppen durch lineare Substitutionen I, Sitzungberichte Königlich Preussischen Akademie Wissenschaften Berlin, 1897, 994-1015.

[8] A.A. Jafarian, A survey of invertibility and spectrum preserving linear maps, Bull. Iran. Math. Soc., 35 (2009), 1-10.

[9] A.A. Jafarian, A.R. Sourour, Spectrum-preserving linear maps, J. Funct. Anal., 66 (1986), 255-266, doi: 10.1016/0022-1236(86)90073-X.

[10] J.P. Kahane and W. Zelazko, A characterization of maximal ideals in commutative Banach algebras, Studia Math., 29 (1968), 339-343.

[11] I. Kaplansky, Algebraic and analytic aspects of operator algebras, In: CBMS Regional Conference series in Math., 1, Amer. Math. Soc. Porvidence, 1970, doi: $10.1090 / \mathrm{cbms} / 001$.

[12] A.M. Gleason, A characterization of maximal ideals, J. Analyse Math., 19 (1967), 171172, doi: 10.1007/BF02788714.

[13] G. Murphy,, $C^{*}$-algebras and Operator Theory, Hardbound, 1990.

[14] M. Omladic, P. Semrl, Spectrum-preserving additive maps, Linear Algebra Appl., 153 (1991), 67-72, doi: 10.1016/0024-3795(91)90210-N. 
[15] T.J. Ransford, A short proof of Johnson's uniqueness-of-norm theorem, Bull. London Math. Soc., 21 (1989), 487-488, doi: 10.1112/blms/21.5.487.

[16] A.R. Sourour, Invertibility preserving linear maps on $L(X)$, Trans. Amer. Math. Soc., 348 (1996), 13-30, doi: 10.1090/S0002-9947-96-01428-6.

[17] W. Zelazko, A characterization of multiplicative linear functionals in complex Banach algebras, Studia Math., 30 (1968), 83-85. 
